\title{
Note to Self: Stop Calling Interfaces "Natural"
}

\author{
Lone Koefoed Hansen \& Peter Dalsgaard \\ Department of Information Studies and Digital Design, \\ Participatory Information Technology, Aarhus University \\ Helsingforsgade 14, Aarhus N, Denmark \\ koefoed@cavi.au.dk, dalsgaard@cavi.au.dk
}

\begin{abstract}
The term "natural" is employed to describe a wide range of novel interactive products and systems, ranging from gesture-based interaction to brain-computer interfaces and in marketing as well as in research. However, this terminology is problematic. It establishes an untenable dichotomy between forms of interaction that are natural and those that are not; it draws upon the positive connotations of the term and conflates the language of research with marketing lingo, often without a clear explanation of why novel interfaces can be considered natural; and it obscures the examination of the details of interaction that ought to be the concern of HCI researchers. We are primarily concerned with identifying the problem, but also propose two steps to remedy it: recognising that the terminology we employ in research has consequences, and unfolding and articulating in more detail the qualities of interfaces that we have hitherto labelled "natural".
\end{abstract}

\section{Author Keywords}

Natural user interfaces; criticism; terminology.

\section{ACM Classification Keywords}

H.5.m. Information interfaces and presentation (e.g., HCI): Miscellaneous.

\section{INTRODUCTION}

The words we use to describe technology matter. They carry with them connotations, establish expectations about the role and use of an object or an interface, and foreground particular qualities, while obscuring others. In many areas of interaction design and HCI, especially the ones pertaining to technical aspects of interaction, rich terminologies are continuously being developed in order to address the intricacies of a particular field. However, in other areas, often those pertaining to the experience and perceived qualities of interfaces, the terminology is disturbingly unnuanced. One instance of this is "natural", a catch-all term that has seen widespread use in industry as well as in academia, both using it as a noun, "natural user interface"

Copyright $(2015$ is held by the author(s). Publication rights licensed to Aarhus University and ACM

5th Decennial Aarhus Conference on Critical Alternatives

August 17 - 21, 2015, Aarhus Denmark

DOI: http://dx.doi.org/10.7146/aahcc.v1i1.21316
(NUI) and a verb, "natural interaction". When viewed from the point of view of interaction design, this is rather strange, given that the experiential qualities of technologies are very often hard to describe; in order to understand and design meaningful interfaces, we need all the help we can get from the words we have at our disposal. Since we in the HCI community evaluate, design, or research relations between a user and a technological setup, we must be better at reminding ourselves that we also need to develop the way that we talk about and characterize the nuances of this interaction.

In this paper, we continue the brief statement in [6] and argue that the use of terms such as "natural" is problematic because the terminology highlights qualities that it does not help us understand and explain adequately, obscuring important aspects at the same time. To frame it in the spirit of the Critical Alternatives conference, we will first offer a critique and then outline alternatives.

\section{“NATURAL” USER INTERFACES}

We specifically wish to criticize the way the term natural is employed to describe user interfaces - an issue that we see not only in marketing, but also in our own work and interaction with colleagues in the field. Even if [13] attempted to dismiss the term in 2010, we nevertheless see university courses on designing for NUIs, workshops at academic conferences with NUI in the title, as well as an increasing number of publications in the ACM Digital Library that explicitly mention NUI (from 19 publications in 2007 to 85 in 2014), of which only few (e.g. [14]) seek to discuss and clarify the use hereof. At present, a wide array of interfaces bears the label. The most common are touch screens, gesture-based interaction, and speech recognition, but the term also encompasses stereo 3D and haptic interfaces [12]. It even extends to specific types of interactions with wellknown instruments, such as the trackpad on a MacBook Air that can be adjusted to offer users a "natural scroll direction" (see Figure 1).

The above list shows that the "naturalness" of an interface is neither defined nor delimited by the underlying technologies, nor does it refer to something that exists in, or emerges from, nature as it is clearly fully man-made and artificial. Rather, the common conception of a natural user interface refers to the experience of interacting with or through the interface, as Microsoft Research Labs' description states "People already use gesture and speech to interact with their PCs and devices; such natural ways to interact with 


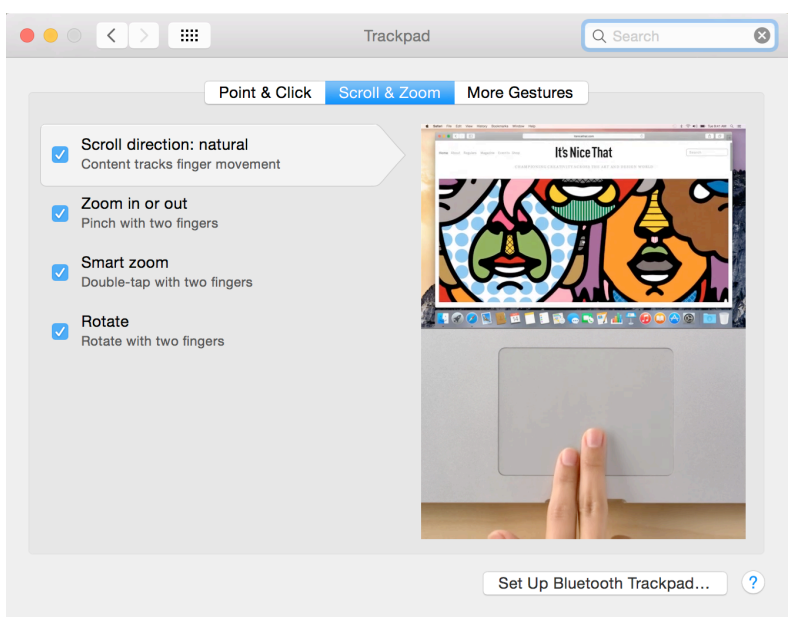

Figure 1: Scroll Direction Tutorial for Apple Trackpad.

with technologies make it easier to learn how to operate them" [9]. On Wikipedia, a NUI is described as "a user interface that is effectively invisible, and remains invisible as the user continuously learns increasingly complex interactions. The word natural is used because most computer interfaces use artificial control devices whose operation has to be learned." In this definition we have at least three different concepts of what "natural" might mean: it is unnoticable ("effectively invisible") since it does not involve a physical ("artificial") input device; it is a walk-up-and-use interface (it doesn't have to be learned); and becoming a super-user is easy. This definition clearly echoes Weiser's vision for the computer for the $21^{\text {st }}$ Century, which would "disappear into the background" [17]; indeed Weiser himself employed metaphors from nature, stating that using computers would become as refreshing "as taking a walk in the woods".

\section{WHY IS IT PROBLEMATIC TO CALL INTERFACES "NATURAL"?}

None of the above readings are intended by those that use the term, obviously, but this only points to a core problem and to our purpose with this paper: words are not only descriptive but also formative - they are important because they help constitute the way we perceive (our possibilities to act in) the world. Central to many language theories is the idea that the world is discursively constructed-we understand the world as well as our possibilities herein also through the way we put it into words. Lakoff's cognitive linguistics is an example of this; in [9] he and Johnson famously highlight how metaphors shape our lived experience of the world. Likewise, Foucault's concept of "discourses" places language as an important part of power structures that change over time, enabling certain developments of society whilst making others less likely [4]. On a micro as well as on a macro level all highlight that it is important to question the terminologies we use. This is done with critique, here understood both in the Frankfurt School tradition of using critical theory to investigate hidden structures, and in the etymological tradition of tracking the meaning of a particular word through time in order to understand its connections to other words.

\section{Naturalized representations}

An important part of Foucault's discourse theory is that a discourse is practically invisible in the period in which it exists - it is often naturalized. In a Foucauldian sense, the dominant discourse of a given period (which can extend over hundreds of years) makes it hard to recognize that the way the world is framed and experienced could be in any other way than it is. Though operating on a smaller scale, French semiotician Roland Barthes' concept of the naturalized connotation is similar; it describes when a connotation, i.e. a representation or understanding of something, is mistaken for a denotation, i.e. a fact, a given, a natural occurence [1]. What Barthes saw was that this communication strategy is used widely in politics and advertising, and he argued that a central aspect of being a modern citizen is to be a skilled sign-reader, i.e. to learn how to spot and challenge attempts of deception.

These strategies of 'deception' are not always on purpose, though, as becomes clear when we turn to feminist theories and critiques where making naturalizations visible in order to change them is a central goal. With language and discourse analyses as "weapon," the goal is to make visible how particular ideas of gender, minorities, etc. are part of power structures that are deeply embedded in a given culture. "We must be wary of the suggestion that anything is natural," is how feminist philosopher Haslanger summarizes this stand, and although she specifically argues that not everything is a cultural construction, language is not one of those things [6]. Language is understood as an encoder of meanings and norms, for instance when the word "man" both means a person of the male sex and the human race in general. Since terminology can be a barrier to accurate communication, the argument is that words must be chosen very carefully, allowing for greater nuances in our understanding of the world and the possibilities we have for changing it. See [15] for an excellent overview of the intersection of feminist critique and language.

In summary, these examples of discourse critique from other fields than HCI show that a constant focus on representation and language in general and on terminology in particular is necessary if we don't want the words we use to describe the world to prevent us from exploring its potentials fully. Following this, we argue below that the term "natural", when referring to user interfaces, is problematic in at least three different respects, namely in regards to the unclear meaning of the term, the issues that it foregrounds, and even more so the issues that it obscures.

\section{What does "natural" mean?}

The first and arguably most obvious critique to raise is that the meaning of the word natural is unclear and imprecise. Considering the definitions outlined earlier, it is difficult to decipher exactly what the common denominator for natural 
user interfaces is, unless we move to a very abstract level: NUIs require no instruments, they are easy to learn, and they disappear into the background. These are dubious claims for most NUIs. Furthermore, "natural" is being employed in ways that contradict this very broad definition, as exemplified by the "natural scroll direction", which clearly indicates that instruments do exist and that there are certain "natural" ways of using them, although, ironically, this way needs to be demonstrated in a tutorial.

In many NUIs, instruments are quite clearly still in play: a gesture based interface such as the Leap Motion controller may obviate the need for a mouse, but it is still clearly present as an instrument that requires the user to maneuver the hands in a specific manner within a constrained interaction zone; likewise, speech recognition may obviate the need for a keyboard, but it still needs to be activated and deactivated in a specific manner, e.g. by picking up a phone and uttering particular activation commands, be it "OK Google" or the anthropomorphic counterpart "Siri". In continuation, these technologies have not eliminated the need for learning how to interact, but rather introduced new interaction modes for us to learn. We need to learn how to do micro-gestures that can be sensed with a reasonable level of precision by the Leap Motion controller; we need to learn how to speak in a monotonous, robotic voice (preferably without accents) to minimize speech recognition errors. In short, we need to learn how to use our bodies as instruments in new ways to accommodate the new "natural" interfaces. Thus, neither the claim of getting rid of instruments nor the claim of not having to learn them seems to hold true. Furthermore, the broad use of the term natural user interface makes "user" a generic concept, implying that some users are more (un)natural than others - as when a Kinect makes it impossible to operate a natural interface when missing an arm or sitting in a wheelchair.

Looking beyond these murky definitions of what makes an interface natural, there also appears to be an underlying assumption that the use of instruments is unnatural, or at least that some instruments are more natural than others. These assumptions, too, are problematic. Firstly, they run counter to the study of human evolution [as discussed in e.g. 5], which indicates that the use of instruments is a crucial aspect of human nature. Secondly, the distinction between more or less natural interfaces is questionable and potentially untenable; e.g. touch and gesture interfaces are referred to as natural user interfaces, but it is unclear how a four-finger swipe is a more natural way of switching to a new desktop space than moving a mouse pointer to a hotspot corner; or how it is more natural to wave your arms in front of a camera mounted on a tv screen in order to calibrate camera tracking so that you can control an in-game cartoon avatar than using a game controller? Thirdly, they ignore the body of work in HCI and beyond that addresses how the use of instruments becomes internalized into human action [e.g. 3]. In summary, the definition of natural in the realm of interaction design and HCI is unclear and imprecise at best, and self-contradictory at worst.

\section{What does "natural" foreground, what does it obscure, and why does it matter?}

Employing discourse analysis to this field, it is clear that the term "natural" is not neutral; rather, it bears with it primarily positive and desirable connotations. Weiser's walk in the woods is "refreshing", the Leap Motion "senses how you naturally move your hands [so that you can do] things you never dreamed possible", [10] and NUIs are "more intuitive, engaging and captivating" [12:1]. From a pragmatic point of view, the desire to label interfaces as natural is understandable. Owing to the imprecise definition of the term, which easily accommodates new technologies as they emerge, this is a non-committal way of imbuing a novel and unfamiliar product with positive associations. Especially in a marketing discourse, "natural" handily uses the naturalization trick that Barthes talked about, marking objects as inescapably good; who wants something unnatural if you can get the natural? However, this approach is unsuitable in a research discourse, where-following Barthes - a central aspect of being a highly skilled and reflective researcher is to be a highly skilled reader and critic of signs, given that words shape the way we give agency to the world.

By using as biased and imprecise a term as "natural", there is a risk of conflating these two very different discourses in HCI, which makes it particularly pertinent for researchers to strive for terminological precision - a prerequisite for the development of a research field. Precision prompts designers to go beyond the surface of a phenomenon, examining and articulating in greater detail how and why it unfolds as it does. Further, precision makes arguments presented in a research community contestable and opens them to joint elaboration, discussion, and development — an essential part of a research field.

While this may appear to be terminological nit-picking, we argue that the words we use matter, even if we are more interested in developing technologies than in discussing semantics. Words have real consequences for how we understand the interfaces we make; for how well we can convey what they do and why to the others; and for how we as a field of both researchers and practitioners can work together to further develop them. The problem with using a term such as natural is that it emphasizes purportedly positive qualities of an interface, but it does not help us understand if, how, and why the interface works. If we are interested in anything beyond promoting a novel interface-and as HCI researchers and practitioners, we ought to be-then we need a better vocabulary to understand, discuss, criticise, and develop these interfaces.

\section{IMPLICATIONS}

Changing a discourse is difficult, but that should not keep us from proposing alternative courses of action, especially in a field that transforms as rapidly as HCI. We consider 
two intertwined steps towards addressing the over-reliance on the term "natural". The first step is to raise awareness that this is in fact problematic. We have laid out a series of criticisms here, from theoretically grounded arguments to more practically oriented ones. While we consider it highly relevant to discuss this topic in the context of the Critical Alternatives conference, we need to bring these criticisms to a wider HCI audience, including venues that might be less amenable to them, if we are to have any hope of having an impact. For this to be constructive, it must be combined with the second step, namely to develop a more refined vocabulary for articulating and addressing what is currently covered by the umbrella term "natural".

The types of interfaces currently labelled natural are being developed and adopted at a rapid pace, and we need to also develop and adapt a language for interfaces able to match the complexity of them. We propose that this can be inspired and informed by recent contributions seeking to unfold the notion of "intuitive interfaces". As with natural, intuitive is a term laden with positive connotations and attached to many an interface, but also one that in itself does not say much about the qualities of an interface and its use. However, recent contributions, including $[2 ; 8 ; 16]$ have examined in more depth what intuitive interaction might mean, and in turn what can make an interface intuitive. At an overarching level, [2] examines properties how prior cultural, sensori-motor, and acquired knowledge influence what is perceived as intuitive; in an attempt to develop tools for examining and refining specific interfaces, [16] has developed a questionnaire for studying if and how users of a given interface conceive of it as being intuitive; and [8] examines what intuitive interaction means in installations in public spaces, drawing among others on the work of [2]. These contributions highlight complementary approaches to developing a more extensive vocabulary of interaction in ways that are theoretically well founded as well as of value to HCI practitioners. since a richer understanding of the notion of intuitive interaction can in turn help developers create better products. The study of the so-called natural user interfaces is in need of similar contributions.

\section{CONCLUSION}

Academic fields outside of HCI have long-standing traditions of discussing the terminology applied within the field itself. Barthes is famous for pointing out how many of the things we say and do are ideologies in camouflage, altough often unintendedly so. This is akin to Luhmann's notion of the blind spot, as well as Marxist and feminist cultural critiques, all focusing on how the way we talk about things shapes our perception of and actions in the world. In this paper, we pursue a similar line of critique in order to examine the notion of "natural" user interfaces. We have argued that the term "natural" does not at all suffice for articulating and understanding these types of interfaces; the use of the term is unnuanced and marred by imprecision and contradictions. Our objective is not to critique the development of novel interfaces; on the opposite, we are advocating the development of a discourse that is rich enough to address the intricacies of these interfaces.

\section{REFERENCES}

1. Barthes, R. 1993 [1957], Mythologies. Vintage, London.

2. Blackler, A.L. \& Hurtienne, J. 2007, Towards a unified view of intuitive interaction: definitions, models and tools across the world. MMI-Interaktiv, 13. pp. 36-54.

3. Bødker, S. Through the interface: A human activity approach to user interface design. Hillsdale, NJ, Lawrence Erlbaum, 1991.

4. Foucault, M. 2001 [1966] The order of things: an archaeology of the human sciences, Routledge.

5. Gibson, K.R. \& Ingold, T. (eds.) 1993, Tools, Language, and Cognition in Human Evolution, Cambridge University Press.

6. Hansen, L.K. 2014, What's in a word?: Why natural isn't objectively better, Interactions, 21, 1, pp. 22-23.

7. Haslanger, S. 2000, Feminism in metaphysics: Negotiating the natural., in Hornsby \& Fricker (eds.), The Cambridge companion to feminism in philosophy, Cambridge University Press.

8. Hespanhol, L. \& Tomitsch, M. 2015, Strategies for Intuitive Interaction in Public Urban Spaces, Interacting with Computers 2015.

9. Lakoff, G. and Johnson, M. 1980, Metaphors we live by. University of Chicago Press, Chicago.

10. Leap Motion. "Leap Motion, Product". http://leapmotion.com/product, accessed 2015-02-16.

11. Microsoft Research "NUI: Natural User Interface", http://research.microsoft.com/en-us/collaboration/ focus/nui/default.aspx, accessed 2015-02-16

12. Murphy, S. 2013, White Paper: Design Considerations for a Natural User Interface (NUI). Texas Instruments.

13. Norman, D. 2010, Natural user interfaces are not natural. interactions 17,3 .

14. O'hara, K., Harper, R., Mentis, H., Sellen, A. and Taylor, A. 2013, On the naturalness of touchless: Putting the "interaction" back into NUI. ACM Trans. Comput.-

Hum. Interact. 20, 1, Article 5.

15. Saul, J. 2012, 'Feminist Philosophy of Language', in Zalta, E. N. (ed.) The Stanford Encyclopedia of Philosophy (Winter 2012 Edition),

16. Ullrich, D. \& Diefenbach, S. 2010, INTUI. Exploring the Facets of Intuitive Interaction. In J. Ziegler \& A. Schmidt (Eds.) Mensch \& Computer 2010 (pp. 251260). München: Oldenbourg.

17. Weiser, M. 1991, The computer for the 21 st century. Scientific American, Sept. 1991, pp. 94-104. 